\title{
Post-Higgs THDM scenarios associated with the new diphoton resonance data
}

\author{
Mikhail Dubinin ${ }^{1,2, \star}$ and Elena Petrova ${ }^{1,2, \star \star}$ \\ ${ }^{1}$ Skobeltsyn Institute of Nuclear Physics, Lomonosov Moscow State University, 119991 Moscow, Russia \\ ${ }^{2}$ Physics Department, Lomonosov Moscow State University, 119991 Moscow, Russia
}

\begin{abstract}
The $C P$-even and $C P$-odd scalar states of the two-Higgs-doublet model (THDM) and the Higgs sector of the Minimal Supersymmetric Standard Model (MSSM), with properties which could lead to the $750 \mathrm{GeV}$ excess in the $\gamma \gamma$ invariant mass distribution, are studied. It is shown that THDM and MSSM are viable in explaining the observed excess without additional assumptions only when the alignment and decoupling limits break down. If the origin of the excess is not in a Higgs sector, regions of the MSSM parameter space are separated where the lightest $C P$-even Higgs boson is identical to the Standard Model scalar.
\end{abstract}

\section{Introduction}

Most significant deviation in the diphoton invariant mass spectrum at $M(\gamma \gamma) \sim 750 \mathrm{GeV}$ observed by ATLAS (integrated luminosity $\left.3.2 \mathrm{fb}^{-1}\right)$ and CMS $\left(2.6 \mathrm{fb}^{-1}\right)$ collaborations in $p p$ collisions at $\sqrt{s}=13 \mathrm{TeV}$ [1] deserves an interpretation in models with an extended Higgs sector. The local excess is about $4 \sigma$, however, the 'look elsewhere effect' reduces the global statistical signiffcance by about $1 \sigma[2]$. Although the excess may be a statistical fluctuation or misidentification of a hadronic state [3] in an absence of other hints of new physics we will treat it as a real physics signal from a new particle denoted as $\Phi$.

Specific properties of the signal rule out the option that it comes from the decay of a spin-1 particle by virtue of the Landau-Yang theorem [4]. A spin 2 graviton is disfavoured because it couples universally to the conserved energy momentum tensor, but no peak is seen in lepton distributions. Bound states with spin 2 which have nothing to do with gravity can couple differently to different particles, and can have odd parity, different angular distributions, which are as motivated as spin 3 bound states [2]. Furthermore, the resonance should be Higgs-like and couple only very weakly to light quarks as, if produced in $q \bar{q}$ annihilation, it should have already been observed at the first LHC run with $\sqrt{s}=8 \mathrm{TeV}$ and $20 \mathrm{fb}^{-1}$ data. We focus here on the more likely possibility of spin zero.

The most natural extension of the Standard Model (SM) is a $C P$-conserving two-Higgs doublet model (THDM), where two Higgs doublets provide five physical states: two $C P$-even neutral Higgs bosons $h$ and $H$, one $C P$-odd neutral Higgs boson $A$ and two charged scalars $H^{ \pm}$, whose masses are free parameters. We suppose that the light $C P$-even state $h$ is identical to one observed with mass 125

${ }^{\star}$ e-mail: dubinin@theory.sinp.msu.ru

$\star \star$ e-mail: petrova@theory.sinp.msu.ru 
$\mathrm{GeV}$ [5]. In order to make in a natural way the lightest $C P$-even state $h$ SM-like, one can invoke the "alignment limit" [6] in which the two mixing angles of scalar states satisfy the condition $\beta-\alpha=\pi / 2$. The $h$ couplings to up and down type fermions and gauge bosons, normalized to the SM values, are then close to one, $g_{h V V} \approx g_{h u u} \approx g_{h d d} \approx 1$, while the couplings of the $C P$-even $H$ and charged $H^{ \pm}$ states reduce to those of the pseudoscalar $A, g_{A V V}=0$.

A particular case of THDM is the Minimal Supersymmetric Standard Model (MSSM) with Yukawa sector of type II, in which very strong constraints are imposed on model parameters at the tree level. We assume that large radiative corrections from the third generation quarks and quarks superpartners play an important role in generation of the effective two-doublet Higgs potential at the scale below the superpartner mass scale $M_{S}$. Besides the alignment limit a favored regime in the MSSM in the context of modern experimental data is the decoupling limit, $\cos (\beta-\alpha) \approx 0$, when $m_{A} \gg m_{Z}$, $m_{H} \approx m_{A} \approx m_{H^{ \pm}}$, in a widely discussed region of low $\tan \beta[7]\left(\tan \beta=v_{2} / v_{1}\right.$, where $\sqrt{v_{1}^{2}+v_{2}^{2}}=246$ $\mathrm{GeV})$.

In an extended scalar sector different possibilities exist for the $\Phi$ particle, which can be either from an isospin singlet state, or from an isospin doublet. Although supersymmetric possibilities include such interpretations of the diphoton resonance as a sgoldstino [8] or a Higgs boson in the Next-toMinimal SSM (NMSSM) [9] in the following we analyse a possibility of $C P$-even and/or $C P$-odd Higgs boson of the THDM contributing to the $\Phi$ signal.

\section{Cross section estimates}

The excess in the invariant mass distribution at $m_{\gamma \gamma}=750 \mathrm{GeV}$ looks like a resonance with the width $\Gamma$ of about $45 \mathrm{GeV}\left(0.06 M_{\Phi}\right)$ with the cross section estimates [10]

$$
\sigma(p p \rightarrow \gamma \gamma)=\left\{\begin{array}{cc}
6 \pm 3 \mathrm{fb} & (\mathrm{CMS}) \\
10 \pm 3 \mathrm{fb} & (\text { ATLAS })
\end{array}\right.
$$

In the following we assume that the excess in the $s$-channel process $p p \rightarrow \Phi \rightarrow \gamma \gamma$ is produced by gluon fusion and subsequent decay to two photons through $t, b$ quarks and $W^{ \pm}, H^{ \pm}$boson loops. The possibilities of large $\tan \beta$ values, $\tan \beta \geq 30$, are excluded for $M_{\Phi}=750 \mathrm{GeV}$ by experimentas searches in the channel $\Phi \rightarrow \tau^{+} \tau^{-}$performed at the run I of the LHC [11], so only small and moderate $\tan \beta$ will be considered in the following evaluations.

In the infinitely small width approximation for the cross section times branching fraction

$$
\sigma(g g \rightarrow \Phi \rightarrow \gamma \gamma)=\sigma(g g \rightarrow \Phi) \times \mathrm{BR}(\Phi \rightarrow \gamma \gamma)
$$

where only main decay channels, $t \bar{t}$ and $b \bar{b}$ (see branching fractions in Fig.1), are taken into account

$$
\mathrm{BR}(\Phi \rightarrow \gamma \gamma) \simeq \frac{\Gamma(\Phi \rightarrow \gamma \gamma)}{\sum_{f=t, b} \Gamma(\Phi \rightarrow f f)}
$$

Then partial widths [12]

$$
\begin{gathered}
\Gamma(\Phi \rightarrow \gamma \gamma)=\frac{G_{\mu} \alpha^{2} M_{\Phi}^{3}}{128 \sqrt{2} \pi^{3}}\left|\sum_{i} A_{i}\left(\tau_{i}\right)\right|^{2}, \\
\Gamma(\Phi \rightarrow f \bar{f})=\frac{3 G_{\mu} m_{f}^{2}}{4 \sqrt{2} \pi} g_{\Phi f f}^{2} M_{\Phi} \beta_{f}^{p_{\Phi}},
\end{gathered}
$$




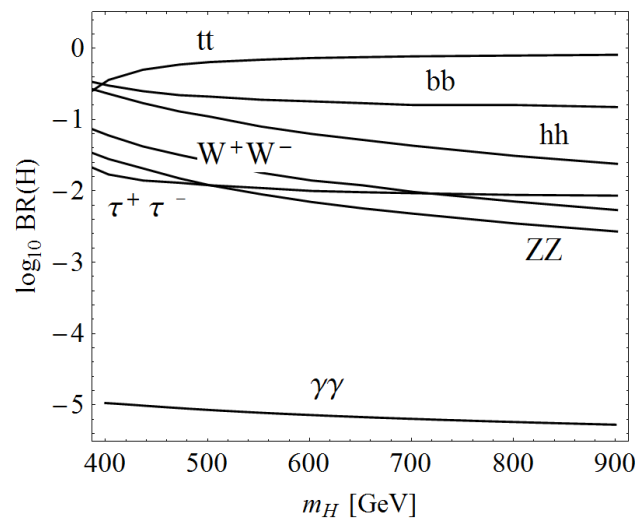

$\mathrm{a}$

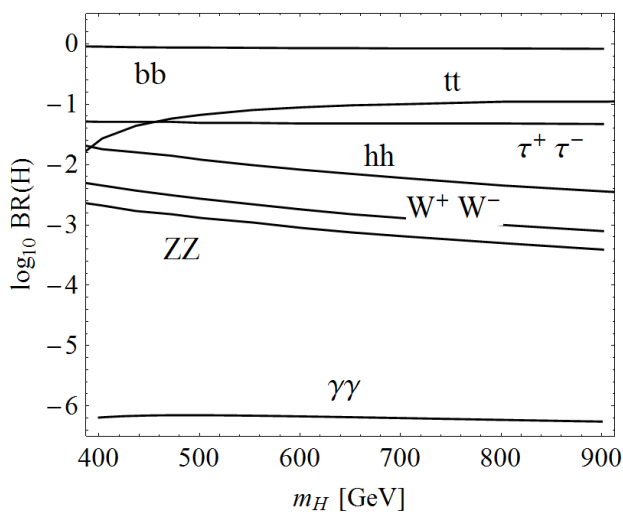

$\mathrm{b}$

Figure 1. MSSM BR(H) in the decoupling limit for $\tan \beta=4$ (a) and $\tan \beta=10$ (b). Here $M_{S}=1.5 \mathrm{TeV}, \mu=1$ $\mathrm{TeV}$ and $\tan \beta=4, A=2.45 M_{S}$ (a); $\tan \beta=10, A=1.5 M_{S}$ (b).

where $\beta_{f}=\left(1-4 m_{f}^{2} / M_{\Phi}^{2}\right)^{1 / 2}$ is the quark velocity and $p_{\Phi}=3$ (1) for the $C P$-even (odd) Higgs boson. The amplitudes of various contributions are

$$
A_{f}=N_{c} Q_{f}^{2} g_{\Phi f f} F_{1 / 2}\left(\tau_{f}\right), \quad A_{W^{ \pm}}=g_{\Phi W^{+} W^{-}} F_{1}\left(\tau_{W^{ \pm}}\right), \quad A_{H^{ \pm}}=g_{\Phi H^{+} H^{-}} \frac{m_{W}^{2}}{m_{H^{ \pm}}^{2}} F_{0}\left(\tau_{H^{ \pm}}\right)
$$

with $1 / 2,1,0$ spin formfactor

$$
\begin{gathered}
F_{1 / 2}^{H}(\tau)=-2[\tau+(\tau-1) f(\tau)] \tau^{-2}, \quad F_{1 / 2}^{A}(\tau)=2 \tau^{-1} f(\tau), \\
F_{0}^{H}(\tau)=[\tau-f(\tau)] \tau^{-2}, \quad F_{1}^{H}=\left[2 \tau^{2}+3 \tau+3(2 \tau-1) f(\tau)\right] \tau^{-2},
\end{gathered}
$$

where $\tau_{f}=M_{\Phi} /\left(4 m_{f}^{2}\right)$ and the function $f(\tau)$ above the kinematical threshold is defined as

$$
f(\tau)=-\frac{1}{4}\left(\log \frac{1+\sqrt{1-\tau^{-1}}}{1-\sqrt{1-\tau^{-1}}}-i \pi\right)^{2}, \quad \tau>1
$$

The production cross section at $\sqrt{s}=13 \mathrm{TeV}$ for $C P$-even and $C P$-odd Higgs bosons of the mass $M_{\Phi}=750 \mathrm{GeV}$ is $\sigma(g g \rightarrow H) \approx 0.85 \cot ^{2} \beta \mathrm{pb}$ and $\sigma(g g \rightarrow A) \approx 1.7 \cot ^{2} \beta \mathrm{pb}$ [13]. More precise calculation containing a combination of fixed order at exact NNLO plus approximate $\mathrm{N}^{3} \mathrm{LO}$ and threshold resummation at $\mathrm{N}^{3} \mathrm{LL}$ [14] does not change the picture significantly [15].

As a rule the cross section value $\sigma(g g \rightarrow H(A) \rightarrow \gamma \gamma)$ is too small $(\sim 0.015 \mathrm{fb}$ for $\tan \beta=1$ in the alignment limit), however the appropriate ranges are still viable for $\tan \beta \simeq 1$ and $\alpha \simeq-0.01$, see Fig.2. In the case of the MSSM, additional contributions provided by supersymmetric particles running in the loops, $\tilde{t}_{i}$ and $\tilde{\chi}_{i}^{ \pm}$, do not change the results. Then the couplings $g_{h V V} \simeq 0.7, g_{h u u} \approx 1.4$, $g_{h d d} \simeq 0.01$ are not SM-like any more. So partial breakdown of the alignment (and decoupling) limit leads to observable value of the THDM (MSSM) production cross section.

On the other hand most explanations of a new resonance at $750 \mathrm{GeV}$ in the alignment limit require the ad-hoc introduction of new, additional particles to the spectrum [13]. The absence of a strong 


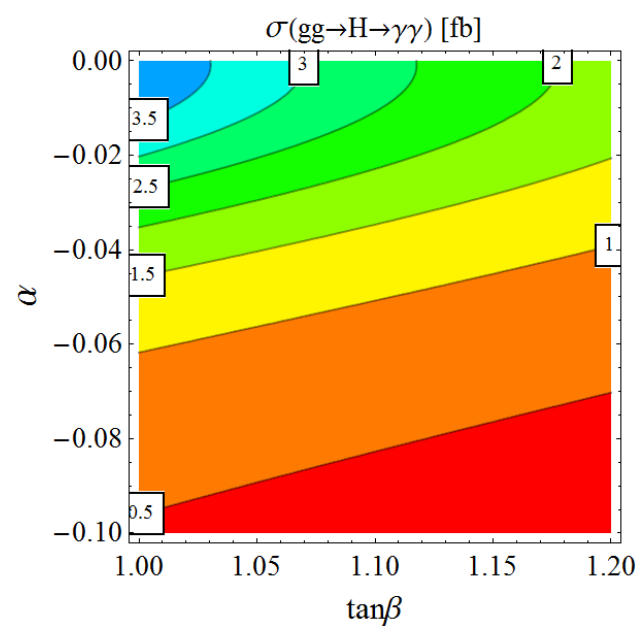

a

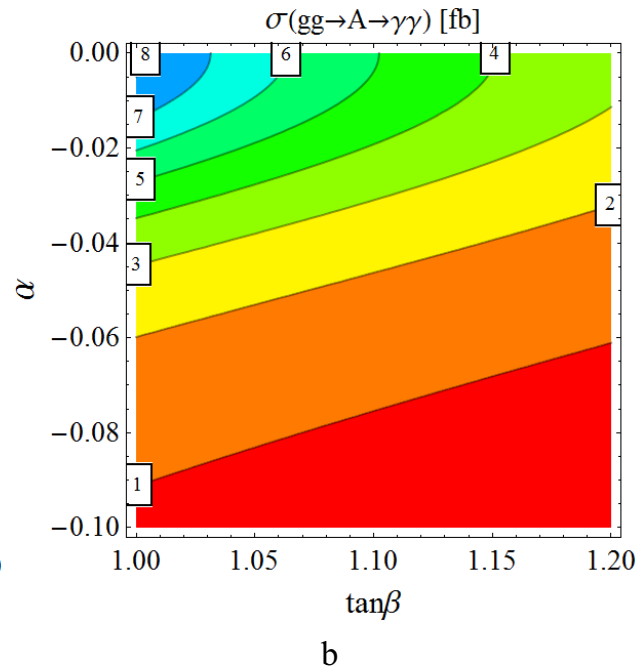

$\mathrm{b}$

Figure 2. The cross section $\sigma(g g \rightarrow \Phi \rightarrow \gamma \gamma)$ [fb] for $\Phi=H(\mathrm{a})$ and $\Phi=A(\mathrm{~b})$, where $m_{\Phi}=750 \mathrm{GeV}$ and $m_{H^{ \pm}}=1.07 \mathrm{TeV}$.

signal in the LHC collisions at $8 \mathrm{TeV}$ motivates gluon fusion as the main production mechanism, presumably mediated by massive coloured fermions and/or bosons. Vector-like quarks or leptons strongly enhance the heavy Higgs couplings to photons and eventually gluons, without altering couplings of the already observed $125 \mathrm{GeV}$ state.

\section{Restricting the MSSM parameter freedom}

In contrast to THDM, the MSSM is strongly constrained. In particular, masses and mixing angles are functions dependent on parameters of radiative corrections. The $C P$-even MSSM Higgs boson squared masses can be expressed as $[16,17]$

$$
\begin{gathered}
m_{H, h}^{2}=\frac{1}{2}\left(m_{A}^{2}+m_{Z}^{2}+\Delta \mathcal{M}_{11}^{2}+\Delta \mathcal{M}_{22}^{2} \pm \sqrt{m_{A}^{4}+m_{Z}^{4}-2 m_{A}^{2} m_{Z}^{2} c_{4 \beta}+C}\right), \\
m_{H^{ \pm}}^{2}=m_{W}^{2}+m_{A}^{2}-\frac{v^{2}}{2}\left(\operatorname{Re} \Delta \lambda_{5}-\Delta \lambda_{4}\right),
\end{gathered}
$$

where

$$
C=4 \Delta \mathcal{M}_{12}^{4}+\left(\Delta \mathcal{M}_{11}^{2}-\Delta \mathcal{M}_{22}^{2}\right)^{2}-4\left(m_{A}^{2}+m_{Z}^{2}\right) \Delta \mathcal{M}_{12}^{2} s_{2 \beta}-2\left(m_{A}^{2}-m_{Z}^{2}\right)\left(\Delta \mathcal{M}_{11}^{2}-\Delta \mathcal{M}_{22}^{2}\right) c_{2 \beta}
$$

and $\Delta \mathcal{M}_{i j}^{2}, \Delta \lambda$ are radiative corrections to the mass matrix elements and self-couplings. The identification of $125 \mathrm{GeV}$ Higgs boson with the $C P$-even light boson $h$ defines the squared mass of the $C P$-odd boson $m_{A}^{2}$ (see Eq. (9))

$$
m_{A}^{2}=\frac{m_{h}^{2}\left(C_{1}-m_{h}^{2}\right)+m_{Z}^{2}\left(C_{2}-C_{3}\right)-\Delta \mathcal{M}_{11}^{2} \Delta \mathcal{M}_{22}^{2}+\Delta \mathcal{M}_{12}^{4}}{C_{1}-C_{2}-C_{3}+m_{Z}^{2} c_{2 \beta}^{2}},
$$

where

$$
C_{1}=\Delta \mathcal{M}_{11}^{2}+\Delta \mathcal{M}_{22}^{2}, \quad C_{2}=m_{h}^{2}-\Delta \mathcal{M}_{12}^{2} s_{2 \beta}, \quad C_{3}=\Delta \mathcal{M}_{11}^{2} s_{\beta}^{2}+\Delta \mathcal{M}_{22}^{2} c_{\beta}^{2}
$$




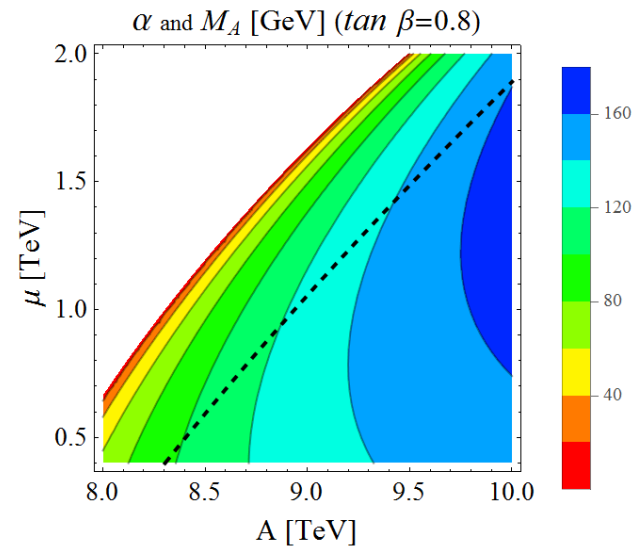

a

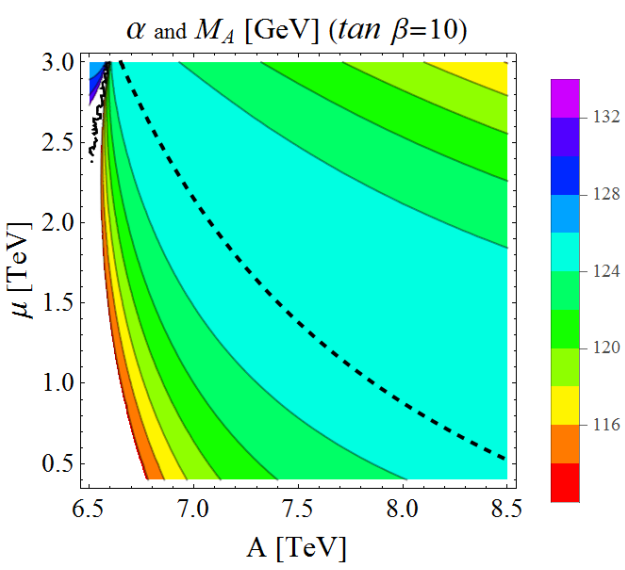

$\mathrm{b}$

Figure 3. $C P$-odd Higgs mass and a requirement $\alpha \approx \beta-\pi / 2$ (dashed black line) in $(A, \mu)$-plane for small (a) and moderate $\tan \beta(\mathrm{b}), M_{S}=2 \mathrm{TeV}$.

Thus all Higgs masses can be unambiguously evaluated in the post-Higgs MSSM. The expression for mixing angle $\alpha$ can be written as [16]

$$
\tan 2 \alpha=\frac{2 \Delta \mathcal{M}_{12}^{2}-\left(m_{Z}^{2}+m_{A}^{2}\right) s_{2 \beta}}{\left(m_{Z}^{2}-m_{A}^{2}\right) c_{2 \beta}+\Delta \mathcal{M}_{11}^{2}-\Delta \mathcal{M}_{22}^{2}} .
$$

A requirement $\alpha \approx \beta-\pi / 2$ and an additional assumption that $m_{\Phi} \sim 1 \mathrm{TeV}$ (in particular, $750 \mathrm{GeV}$ ) result in a rather significant constraints imposed on the MSSM paramer space. The following analysis is based on the calculation of radiative corrections in the MSSM Higgs sector by means of the effective potential method [18], taking into account carefully the nonleading soft supersymmetry breaking $D$ terms, the wave-function renormalization terms and also including the QCD and weak corrections to Yukawa couplings up to two loops [19]. Only top and bottom quarks and their superpartners in a loop are taken into account. In such a scenario the MSSM parameter space has the dimention four - $\tan \beta$, $M_{S}$ and soft-breaking SUSY parameters $A$ (in the general case $A_{t} \neq A_{b}$, for simplicity we assume $\left.A_{t}=A_{b}\right)$ and $\mu$. These parameters respect the conditions

$$
2\left|m_{t} A\right|<M_{S}^{2}, \quad 2\left|m_{t} \mu\right|<M_{S}^{2}
$$

if we restrict the analysis of effective potential by the dimension-four operators. For numerical calculations we consider parameter sets $\left(M_{S}, \tan \beta\right)$, where $M_{S}=1.5,2,5,10 \mathrm{TeV}$ and $\tan \beta=$ $0.8,2,3,10,20$, and check general mass basis conditions for them

$$
m_{h}=125 \mathrm{GeV}, \quad m_{H, A, H^{ \pm}}>0 .
$$

It turns out that the alignment limit $\alpha \approx \beta-\pi / 2$ is satisfied in rather restricted domain of $A$ and $\mu$. With combination of mass basis conditions it resuts in almost degenerate mass spectrum $m_{h}=m_{H}$ and $m_{H} \approx m_{A} \approx m_{H}^{ \pm}$. Characteristic structure is presented in Fig.3 for $M_{S}=2 \mathrm{TeV}$ and $\tan \beta=0.8$ and 10 .

The possibility of $m_{\Phi} \sim 1 \mathrm{TeV}$ breaks the alignment limit. In this case the large $\tan \beta$ region for $M_{S}$ up to $10 \mathrm{TeV}$ is completely excluded. For $M_{S}$ of about a few $\mathrm{TeV}$ the low $\tan \beta$ region is severely 
restricted. As a rule only two couplings $g_{h V V} \approx g_{h u u} \approx 1$ are SM-like. The additional constraint of the $\sigma$ value, see (1), results in unambiguously defined parametric sets $A$ and $\mu$ for fixed $M_{S}$. For example, $A=61.490 \mathrm{TeV}, \mu=19.334 \mathrm{TeV}$ for $M_{S}=5 \mathrm{TeV}$, and for $\tan \beta=1$ the corresponding values are $m_{h}=125 \mathrm{GeV}, m_{H}=750 \mathrm{GeV}, m_{A}=893 \mathrm{GeV}, m_{H^{ \pm}}=1073 \mathrm{GeV}, \alpha=-0.0001$ and $g_{h V V} \simeq 0.7$, $g_{\text {huu }} \simeq 1.4, g_{\text {hdd }} \simeq 0.0001$.

\section{Summary}

A number of estimates for the post-Higgs THDM and the MSSM effective Higgs sector respecting the restrictions on the model parameter space inspired by the observation of the diphoton excess at an invariant mass of about $750 \mathrm{GeV}$ have been considered. If the signal within these models is real and has a Higgs nature it will result in significant consequences for models parameter space. Either the models include the SM-like state $h$ in the approximate alignment limit with additional charged particles introduced $a d-h o c$, or the alignment limit is broken and the state $h$ is not SM-like fully or partially. In any case the allowed parameter space is strongly restricted.

In the case of minimal supersymmetry it follows from the estimates that the SM-like Higgs properties of the observed boson $h$ in the stringent alignment limit exclude completely masses of the MSSM Higgs bosons of about $1 \mathrm{TeV}$ and lead to close to degenerate mass spectrum $m_{h}=m_{H}, m_{H} \approx m_{A} \approx m_{H}^{ \pm}$. Renunciation of this requirement results in satisfactory description of the $C P$-even $h$ and $H$ (or $A$ ) boson mass, width (for $h$ ) and cross section for the $g g$ initial state in the case when there are deviations from the alignment limit and properties of the state $h$ are not fully SM-like.

This work was supported by Russian Science Foundation, grant 16-12-10280.

\section{References}

[1] ATLAS collaboration, ATLAS-CONF-2015-081; ATLAS-CONF-2016-018

[2] A. Strumia, Interpreting the $750 \mathrm{GeV}$ digamma excess: a review, arXiv:1605.09401[hep-ph]

[3] K. Bondarenko et al., Features in the Standard Model diphoton background, arXiv:1606.09592[hep-ph]

[4] L. Landau, Dokl. Akad. Nauk USSR, Ser.Fiz. 60, 207 (1948); C.N. Yang, Phys. Rev. 77, 242 (1950)

[5] G. Aad et al. (ATLAS Collaboration), Phys. Lett. B. 716, 1 (2012); S. Chatrchyan et al. (CMS Collaboration), Phys. Lett. B.716, 30 (2012)

[6] M. Carena et al., Phys. Rev. D 91, 035003 (2015)

[7] E. Bagnashi et al., LHCHXSWG-INT-2015-004

[8] P. Baratella et al., A closer look to the sgoldstino interpretation of the diphoton excess, arXiv:1603.05682v1[hep-ph]; K. O. Astapov, D. S. Gorbunov, Phys. Rev. D 93, 035008 (2016)

[9] S. Heinemeyer, The $750 \mathrm{GeV}$ diphoton excess and SUSY, arXiv:1605.08324v1[hep-ph]

[10] R. Franceschini et al., What is the $\gamma \gamma$ resonance at 750 GeV? arXiv:1512.04933[hep-ph]

[11] ATLAS collaboration, JHEP 11, 056 (2014); CMS collaboration, JHEP 1410, 160 (2014).

[12] A. Djouadi et al., The coupling of the lightest SUSY Higgs boson to two photons in the decoupling regime, arXiv:9612362v1[hep-ph]; A. Djouadi, Phys. Rept. 457, 1-216 (2008); A. Djouadi et al., Fully covering the MSSM Higgs sector at the LHC, arXiv:1502.05653v2[hep-ph]

[13] A. Angelescu, A. Djouadi, Gr. Moreau, Scenarii for interpretations of the LHC diphoton excess: two Higgs doublets and vector-like quarks and leptons, arXiv:1512.04921v2[hep-ph]

[14] T. Ahmed et al., Pseudo-scalar Higgs boson production at $\mathrm{N}^{3} \mathrm{LO}_{A}+\mathrm{N}^{3} \mathrm{LL}^{\prime}$, arXiv:1606.00837v1[hep-ph] 
[15] C. Anastasiou et al., CP-even scalar boson production via gluon fusion at the LHC, arXiv:1605.05761v1[hep-ph]

[16] Y. Okada, M. Yamaguchi, T. Yanagida, Phys. Lett. B 262, 54 (1991); J. Ellis, G. Ridolfi, F. Zwirner, Phys. Lett. B 257, 83 (1991); H.E. Haber, R. Hempfling, Phys. Rev. Lett. 66, 1815 (1991); R. Barbieri, M. Frigeni, F. Caravaglios, Phys. Lett. B 258, 167 (1991)

[17] A. Djouadi et al., Eur. Phys. J. C 73, 2650 (2013); E. Bagnashi et al., LHCHXSWG-INT-2015004

[18] E. Akhmetzyanova, M. Dolgopolov, M. Dubinin, Phys. Rev. D 71, 075008 (2005); Fiz. Elem. Chast. Atom. Yadra 37, 677 (2006)

[19] H. E. Haber, R. Hempfling, A. Hoang, Z. Phys. C 75, 539 (1997); M. Carena, M. Quiros, C.E.M. Wagner, Nucl. Phys. B 461, 407 (1996); S. Heinemeyer, W. Hollik, G. Weiglein, Eur. Phys. J. C 9, 343 (1999); A. Pilaftsis, C.E.M. Wagner, Nucl. Phys.B 553, 3 (1999) 